\title{
EPMA WDS Peak Position Analysis of Mineral Chemistry in Fossils
}

\author{
Douglas C. Meier ${ }^{1,2}$, Kimberly K. Foecke ${ }^{3}$, Edward P. Vicenzi ${ }^{4}$, Peter J. Heaney ${ }^{3}$, and Russell Graham ${ }^{3}$ \\ 1. McCrone Associates, Inc., Electron Optics Group, Westmont, IL, USA. \\ 2. National Institute of Standards and Technology, Material Measurement Science Division, \\ Gaithersburg, MD, USA. \\ 3. The Pennsylvania State University, Department of Geosciences, University Park, PA, USA. \\ 4. Smithsonian Institution, Museum Conservation Institute, Suitland, MD, USA.
}

Frequently in EPMA, the chemistry of an analyte (that is, the oxidation state and bonding of the elements in the material) is inferred from the composition, by stoichiometry or some other means. While this practice is usually adequate for determining the identity of pure single-phase materials, the results are often inconclusive when attempting similar analyses of complex mixtures composed of multiple compounds and phases. However, there are photoemission processes for certain elements in which a valence electron fills an atom's core vacancy, resulting in characteristic X-rays that differ slightly in wavelength from the diagram line. In these circumstances, the peak position can be exploited by the analyst to determine an element's oxidation state within the matrix. A practical problem, identifying sulfur mineralization within fossilized bones, is presented to demonstrate the method. The sulfur $K$ lines have been shown to exhibit peak positions dependent upon oxidation state [1,2].

Fossilized mammoth teeth from the Pleistocene epoch were collected from peat-like sediments at the bottom of the Santa Fe River in Florida, where they had been periodically partially buried and exposed to river water. They exhibit post-depositional alterations that are typical of such specimens independent of geographic origin. Chemical analyses of bones such as these are used routinely in paleoenvironmental and dietary reconstructions for the time at which the animal died. These measurements rely heavily on precise quantitative elemental and isotopic data. Post-depositional chemical alterations on the microscale are poorly understood, yet could have large effects on these types of reconstructions. To ensure the accuracy of paleo-reconstruction measurements, micro-scale taphonomic effects must be understood and fully characterized so that corrections for data analysis can be developed.

Conventional EPMA analyses were performed on mounted, polished, and carbon-coated tooth specimens. Calcium, phosphorous, and oxygen consistent with hydroxyapatite were found, as were iron and sulfur. Reference materials for sulfate (gypsum), sulfide (troilite) and elemental sulfur were analyzed to determine their respective peak centers (Figure 1). These peak position reference materials were analyzed using wavelength spectrometers tuned to both the sulfate and sulfide peak centers, and relative intensity ratios were determined. The tooth specimens were then compositionally mapped using WDS; both the sulfate and sulfide peak positions were measured simultaneously. The sulfate/sulfide intensity ratios of the specimen were compared to the references in order to estimate the positiondependent chemical state of the sulfur in the specimen. Sulfide was found to correlate most strongly to those locations where the sulfur concentration was highest (Figure 2), but not as strongly to the locations with the highest iron concentration (not shown). Furthermore, pure sulfide (red) is indicated in only a few positions throughout the sample, suggesting that elemental sulfur or sulfate may also be present. Thus, while pyrite is likely the dominant sulfur-bearing mineral in this fossil, other iron-bearing species and trace sulfate (possibly as a phosphate substitution) are also indicated in the specimen. 


\section{References:}

[1] P D Pérez, A C Carreras and J C Trincavelli, J. Phys. B: At. Mol. Opt. Phys. 45 (2012) 025004.

[2] M R Carroll and M J Rutherford, Am. Min., 73 (1988) 845-849.

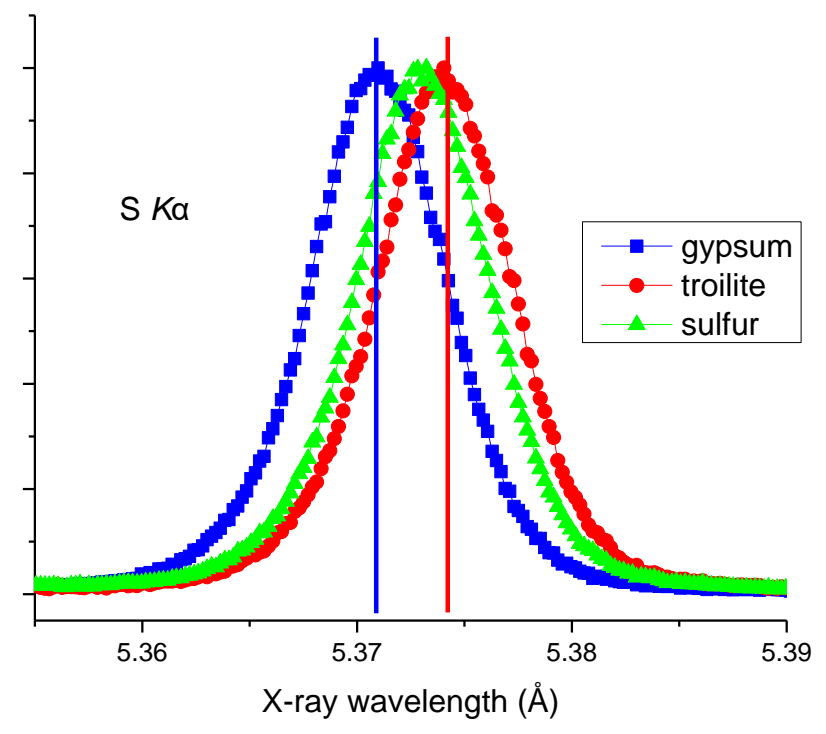

Figure 1. Normalized WDS wavelength scans of a sulfate (gypsum, nominal sulfur oxidation state 6+), a sulfide (troilite, sulfide ion oxidation state 2-), and sulfur (oxidation state 0).

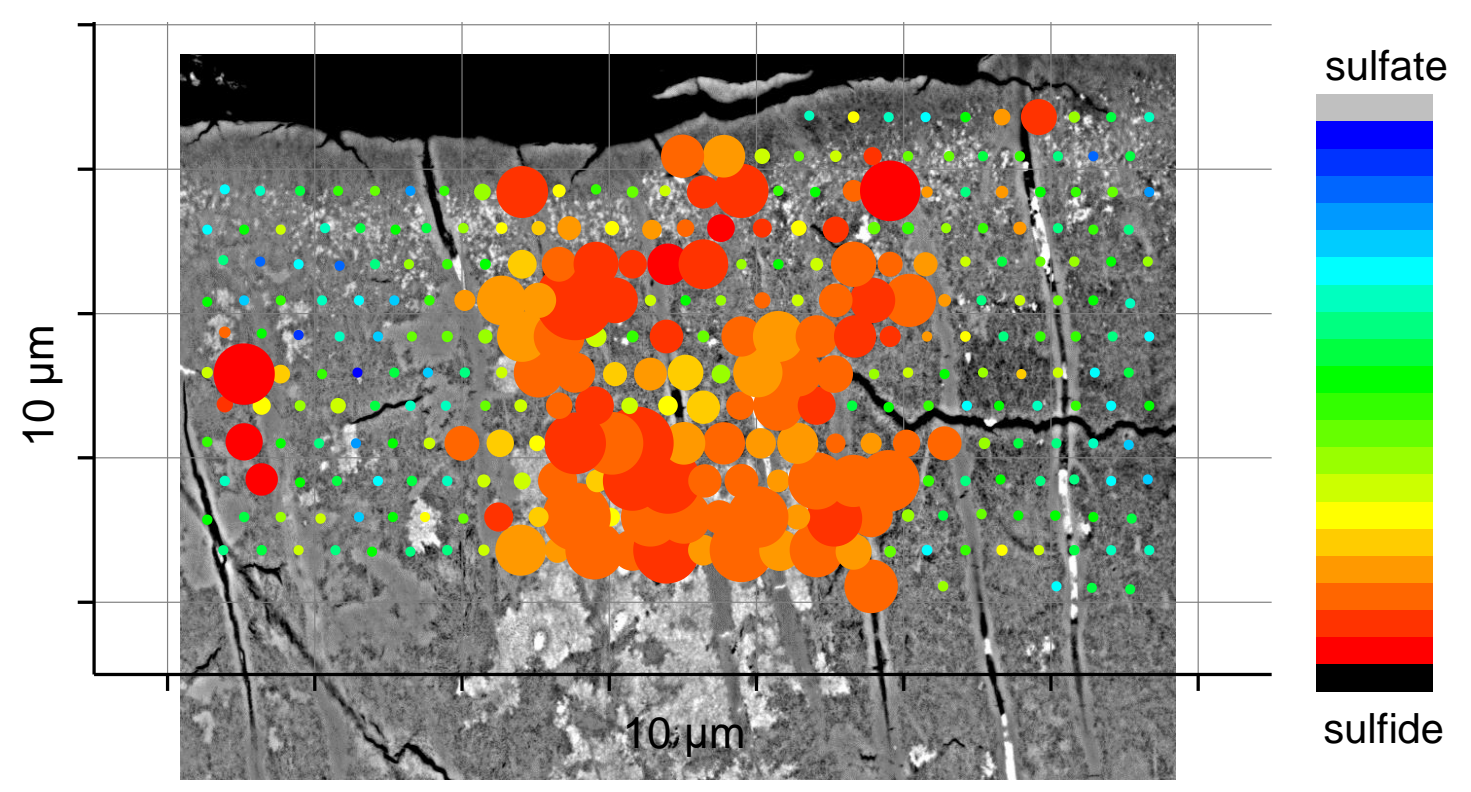

Figure 2. EPMA WDS analysis bubble map grid superimposed over an image of the fossilized horse tooth specimen. The color of the bubble corresponds to mean oxidation state at that analysis point, while the size of the bubble corresponds to the sulfur concentration at that point, ranging from $0.04 \%$ to $14 \%$ mass fraction. 